\title{
Calibration of a Camera-Based Guidance Solution for Orthopedic and Trauma Surgery
}

\author{
Jessica Magaraggia ${ }^{1}$, Adrian Egli ${ }^{2}$, Gerhard Kleinszig ${ }^{2}$, Rainer Graumann², \\ Elli Angelopoulou ${ }^{1}$, Joachim Hornegger ${ }^{1}$ \\ ${ }^{1}$ Pattern Recognition Lab, University of Erlangen-Nuremberg, Erlangen, Germany \\ ${ }^{2}$ Siemens AG, Healthcare Sector, Erlangen, Germany \\ jessica.magaraggia@informatik.uni-erlangen.de
}

\begin{abstract}
In orthopedic and trauma surgery, fracture reduction usually requires the use of metallic plates and their fixation by means of screws. The employment of guidance solutions during surgical procedures has become of great importance during the last decades. Our guidance solution exploits a small video camera placed directly on the instrument, for example a drill, and a set of small markers placed around the location where the drilling needs to be performed. A calibration step is required in order to determine the relative position of the instrument tip and axis w.r.t the coordinate system of the video camera. In this paper we describe a calibration method for our guidance solution. This calibration method exploits optical markers and a calibration plate whose geometry is known. Moreover, we show how we can exploit directly the image acquired by the video camera during the calibration in order to define an error measure to estimate the accuracy of the calibration. With this method, we achieved respectively an accuracy of $0.23 \mathrm{~mm}$ and $3.40^{\circ}$ in the estimation of the instrument tip position and of the orientation of the instrument axis.
\end{abstract}

\section{Introduction}

In orthopedic and trauma surgery, guidance solutions are often exploited in order to help the surgeon fixing screws during a fracture reduction procedure. Typically, optical navigation solutions (e.g. VectorVision ${ }^{\circledR}$ from BRAINLAB AG. and Navigation System II from Stryker Inc.) use a stereo camera placed outside the operative field $[1,2]$. Solutions based on video cameras have also been proposed. Diotte et al. [3], for example, exploited an augmented reality C-arm and a modified Schanz screw to determine the tip position of the drilling guide during the procedure by means of video images. Real-time display of its position is then possible on both video and X-ray images since they are coregistered after calibration of the device. Our proposed solution ${ }^{1}$ does not require an augmented C-arm. We exploit a small video camera placed on the surgical instrument, e.g.

\footnotetext{
${ }^{1}$ The presented method is investigational use and is limited to investigational use. It is not commercially available and its future availability cannot be ensured
} 
a surgical drill. To our purpose, a calibration step is required in order to relate the position of the axis and of the tip of the instrument to the camera. After the calibration, provided that optical markers are positioned where the instrument has to be inserted, it will be possible to exploit the images acquired from the video camera to determine the position of the attached instrument in realtime. Position feedback could be therefore provided to the surgeon during the procedure. A similar video camera based approach has been proposed by the medical company ActiViews, Inc. for percutaneous lung and liver interventions under CT-guidance. Their approach exploits the standard design of percutaneous instrumentation, such as biopsy needles and ablation probes that allows a camera-instrument calibration just relative to the individual instrument length. In comparison, we need to provide a full instrument calibration since we need to calibrate both tip position and axis direction of the instrument. In the following sections, we describe our calibration setup and the steps of the calibration procedure. Moreover, we propose a method for the estimation of the calibration error based directly on the images acquired from the video camera attached to the instrument.

\section{Materials and Methods}

Our calibration setup is illustrated in Fig. 1. The calibration tool is made of a holder plate with an insertion guide that hosts the instrument axis and that is orthogonal to the plate's surface. Around its entry location, on the surface of the calibration plate, square optical markers are placed. The markers are similar to the ones proposed by Forman et al. [4]. The center of the entry location of the insertion guide is chosen to be the origin of our reference coordinate system $M$. The $z$-axis of $M$ is also orthogonal to the plate surface (see Fig. 1(a)). The user has to position the instrument so that its axis is inserted into the guide and the tip reaches its bottom. The tip position is then defined by $\mathbf{P}_{\mathbf{M}}=(0,0,-d)^{T}$, where $d$ is the depth of the guide, and the axis direction by $\mathbf{v}_{\mathbf{M}}=(0,0,1)^{T}$, w.r.t $M$. The geometry of the markers is also known w.r.t $M$. As a consequence, the transformation matrix $\mathbf{T}_{\mathbf{C}}^{\mathbf{M}}$ from $M$ to the camera coordinate system $C$ can be determined exploiting a set of known point correspondences. The latter are defined between the corners of the markers in $M$ and their corresponding points location in the images acquired by the camera. For the estimation of $\mathbf{T}_{\mathbf{C}}^{\mathbf{M}}$ we exploit the Perspective-n Points algorithm from Schweighofer et al. [5].

Once $\mathbf{T}_{\mathbf{C}}^{\mathbf{M}}$ is known, we can easily describe our tool position in the camera coordinate system as $D_{\mathrm{C}}=\left(\mathbf{P}_{\mathbf{C}}, \mathbf{v}_{\mathbf{C}}\right)$ where $\mathbf{P}_{\mathbf{C}}$ and $\mathbf{v}_{\mathbf{C}}$ are respectively the position of the instrument tip and axis orientation expressed in $C$ :

$$
\left[\begin{array}{c}
\mathbf{P}_{\mathbf{C}} \\
1
\end{array}\right]=\mathbf{T}_{\mathbf{C}}^{\mathbf{M}}\left[\begin{array}{c}
\mathbf{P}_{\mathbf{M}} \\
1
\end{array}\right] \quad\left[\begin{array}{c}
\mathbf{v}_{\mathbf{C}} \\
1
\end{array}\right]=\mathbf{T}_{\mathbf{C}}^{\mathbf{M}}\left[\begin{array}{c}
\mathbf{v}_{\mathbf{M}} \\
1
\end{array}\right]
$$

One camera acquisition could suffice for the estimation of $D_{\mathrm{C}}$. However, the accuracy of our estimation depends on the validity of our hypothesis that the 


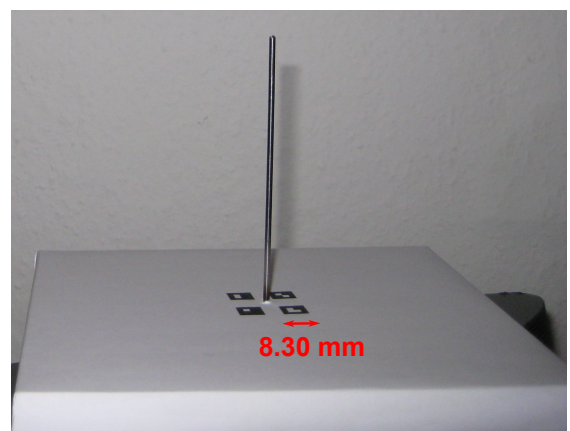

(a)

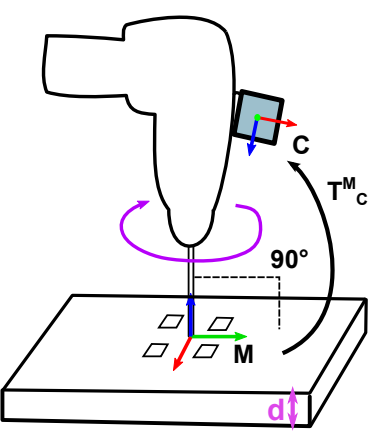

(b)

Fig. 1. Our calibration plate (a). Complete calibration setup (b).

instrument axis remains orthogonal to the calibration plate and that the instrument tip does not move. If small deviations of the instrument position occur, $\mathbf{v}_{\mathbf{M}}$ and $\mathbf{P}_{\mathbf{M}}$ no longer represent the correct axis orientation and tip position w.r.t $M$. Such deviations could occur naturally as a consequence of the instrument weight or could be induced by the user while holding the instrument. In order to reduce the influence of small deviations of the instrument position, we perform a set of measurements rotating the upper part of the instrument of approximately $360^{\circ}$ about its axis $\mathbf{v}_{\mathbf{M}}$. While rotating, the camera is continuously acquiring and for each frame, the relation described in Eq. 1 can be established. In the end, all measurements are combined together as described in the following in order to achieve a more robust estimation of $D_{\mathrm{C}}$.

First of all, we observe that the instrument axis and tip are inside the field of view of the camera. Since the relative position between the camera and the instrument axis is fixed by construction, its position in the camera image does not change. Before starting the calibration procedure, we acquire an image $I_{\mathrm{G}}$ from our camera on a white background. From this image, we determine the tip position $\mathbf{I}_{\mathbf{P}}=\left(x_{\mathrm{P}}, y_{\mathrm{P}}\right)^{T}$ and the axis orientation $\mathbf{v}_{\mathbf{P}}=\left(v_{\mathrm{X}}, v_{\mathrm{Y}}\right)^{T}$ in the image coordinate system as shown in Fig. 3. $\mathbf{I}_{\mathbf{P}}$ and $\mathbf{v}_{\mathbf{P}}$ can be used as ground truth in order to obtain a measurement of the accuracy of our estimation.

For each image frame $i$, we get an estimation of $\mathbf{P}_{\mathbf{C}}^{\mathbf{i}}$ and $\mathbf{v}_{\mathbf{C}}^{\mathbf{i}}$. Since we calibrated our camera, we can exploit the intrinsic matrix and the distortion coefficients estimated during the camera calibration to project $\mathbf{P}_{\mathbf{C}}^{\mathbf{i}}$ and $\mathbf{A}_{\mathbf{C}}^{\mathbf{i}}=\mathbf{P}_{\mathbf{C}}^{\mathbf{i}}+\mathbf{v}_{\mathbf{C}}^{\mathbf{i}}$ onto the image plane [6]. After, $\mathbf{I}_{\mathbf{C}}^{\mathbf{i}}$ is calculated as the corresponding image tip of $\mathbf{P}_{\mathbf{C}}^{\mathbf{i}}$. Since $\mathbf{A}_{\mathbf{C}}^{\mathbf{i}}$ represents a point on the instrument axis, we calculate its correspondent image point $\mathbf{I}_{\mathbf{A}_{\mathbf{C}}}^{\mathbf{i}}$. The vector $\mathbf{v}_{\mathbf{I}}^{\mathbf{i}}=\mathbf{I}_{\mathbf{A}_{\mathbf{C}}}^{\mathbf{i}}-\mathbf{I}_{\mathbf{C}}^{\mathbf{i}}$ represents the orientation of the instrument axis in the image. We then define $e_{\mathrm{P}}^{i}$ and $e_{\alpha}^{i}$ as respectively the tip position error and the angular error of the axis in the image by:

$$
e_{\mathrm{P}}^{i}=\left\|\mathbf{I}_{\mathbf{C}}^{\mathbf{i}}-\mathbf{I}_{\mathbf{P}}\right\|_{\mathbf{2}} \quad e_{\alpha}^{i}=\arccos \frac{\mathbf{v}_{\mathbf{I}}^{\mathbf{i}} \cdot \mathbf{v}_{\mathbf{P}}}{\left\|\mathbf{v}_{\mathbf{I}}^{\mathbf{i}}\right\|_{2}\left\|\mathbf{v}_{\mathbf{P}}\right\|_{2}}
$$


An overlay of the tracked tool tip and tracked axis onto 2-D or 3-D X-ray data sets or on video frames acquired from a video camera can be usually performed in order to have a feedback of the accuracy of the instrument position $[2,3]$. However, this overlay requires a registration step between the tracker and the images on which the overlay is performed. In our case, no registration is required since Eq. 2 directly gives a feedback about the calibration accuracy.

We also define the weights $w_{\mathrm{P}}^{i}$ and $w_{\alpha}^{i}$, that are respectively associated to the estimation of the tip position and angular orientation at the frame $i$.

$$
w_{\mathrm{P}}^{i}=1-\frac{e_{\mathrm{P}}^{i}}{\sum_{j=1}^{N} e_{\mathrm{P}}^{j}} \quad w_{\alpha}^{i}=1-\frac{e_{\alpha}^{i}}{\sum_{j=1}^{N} e_{\alpha}^{j}}
$$

According to Eq. 3, a smaller error in the estimation of $\mathbf{P}_{\mathbf{C}}^{\mathbf{i}}$ and $\mathbf{v}_{\mathbf{C}}^{\mathbf{i}}$ is associated respectively with a bigger $w_{\mathrm{P}}^{i}$ and $w_{\alpha}^{i}$. Both weights are used to obtain a final estimation of the tip position $\overline{\mathbf{P}}_{\mathbf{C}}$ and of the axis orientation $\overline{\mathbf{v}}_{\mathbf{C}}$ according to Eq. 4. A position estimation $\mathbf{P}_{\mathbf{C}}^{\mathbf{i}}$ and an angular orientation $\mathbf{v}_{\mathbf{C}}^{\mathbf{i}}$ which returned a small estimation error contribute therefore more to the final estimation of the tip position and of the angular orientation $\overline{\mathbf{P}}_{\mathbf{C}}$ and $\overline{\mathbf{v}}_{\mathbf{C}}$.

$$
\overline{\mathbf{P}}_{\mathbf{C}}=\frac{\sum_{i=1}^{N} w_{\alpha}^{i} \mathbf{P}_{\mathbf{C}}^{\mathbf{i}}}{\sum_{i=1}^{N} w_{\alpha}^{i}} \quad \overline{\mathbf{v}}_{\mathbf{C}}=\frac{\sum_{i=1}^{N} w_{\mathrm{P}}^{i} \mathbf{v}_{\mathbf{C}}^{\mathbf{i}}}{\left\|\sum_{i=1}^{N} w_{\mathrm{P}}^{i} \mathbf{v}_{\mathbf{C}}^{\mathbf{i}}\right\|_{2}}
$$

The instrument in camera coordinate system is then described by:

$$
\bar{D}_{\mathrm{C}}:=\left(\overline{\mathbf{P}}_{\mathbf{C}}, \overline{\mathbf{v}}_{\mathbf{C}}\right)
$$

For the evaluation of our calibration method, we positioned our instrument as depicted in Fig. 1(b) and rotated it of $360^{\circ}$ about its axis $\mathbf{v}_{\mathbf{M}}$. Before starting the rotation, we turned our firewire camera on and continued acquiring while rotating. Our images were acquired uniformly over the whole span range of approximately $360^{\circ}$ with a resolution of $768 \times 1024$ obtaining 422 image-frames. For each frame $i$, we calculated $e_{\mathrm{P}}^{i}$. The tip position that returned the minimum error for the whole sequence, $\mathbf{P}_{\mathbf{C}}^{\mathbf{M i n}}$ and the current $\mathbf{v}_{\mathbf{C}}^{\mathbf{i}}$ were used for calculating $\mathbf{A}_{\mathbf{C}}^{\mathbf{i}}$ in each frame. Using $\mathbf{P}_{\mathbf{C}}^{\mathbf{M i n}}$ instead of the corresponding $\mathbf{P}_{\mathbf{C}}^{\mathbf{i}}$ for each frame allows to investigate the error in the angular orientation independently from the error in the estimation of the tip position. For each frame also the angular error $e_{\alpha}^{i}$ was calculated. After, the whole set of measurements was used to get the final estimation of $\bar{D}_{\mathrm{C}}$ as described in Eq. 4 and Eq. 5.

\section{Results}

The results of our instrument calibration are shown in Fig. 2. The reported errors are calculated according to Eq. 2. As concerning the tip position error, we used an empirically determined conversion factor $1 \mathrm{px}=0.05 \mathrm{~mm}$ in order to express our results in $\mathrm{mm}$. This factor is proportional to the camera-markers distance. In Fig. 2(a) and Fig. 2(c) we show respectively the results of the tip position 
and of the axis orientation estimation evaluated for each frame separately. The position of the instrument tip and the axis orientation could be estimated with an accuracy of $1.39 \pm 0.41 \mathrm{~mm}$ and $3.36 \pm 0.72^{\circ}$. In Fig. 2(b) and Fig. 2(d) we report the errors obtained estimating $\mathbf{P}_{\mathbf{C}}^{\mathbf{i}}$ and $\mathbf{v}_{\mathbf{C}}^{\mathbf{i}}$ for the frame $i$ considering also the previous $i-1$ frames according to Eq. 4 . At the last frame, for which all the 422 frames available are considered, we obtained $e_{\mathrm{P}}^{N}=0.23 \mathrm{~mm}$ and $e_{\alpha}^{N}=$ $3.36^{\circ}$ where $N=422$. The resulting $\mathbf{P}_{\mathbf{C}}^{\mathbf{N}}$ and $\mathbf{v}_{\mathbf{C}}^{\mathbf{N}}$ represent our final estimation $\bar{D}_{\mathrm{C}}$, which reprojected onto the image lead to an angular error of $3.40^{\circ}$. The final result of the calibration is depicted in Fig. 3.

\section{Discussion}

In this paper we presented a calibration method for a camera-based guidance solution. The method allows to determine the instrument position and the instrument axis direction with an accuracy of $0.23 \mathrm{~mm}$ and $3.40^{\circ}$ respectively. Optical

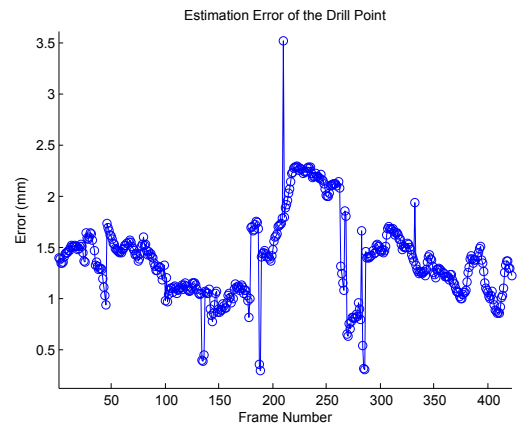

(a)

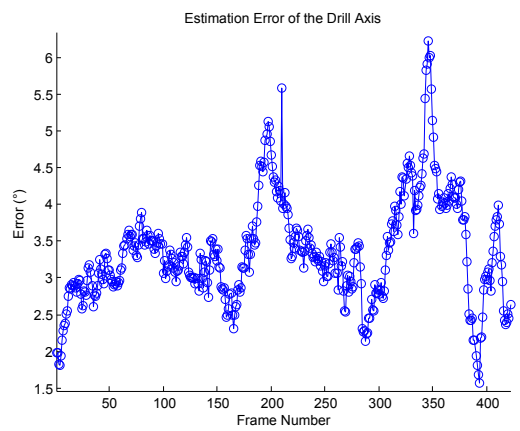

(c)

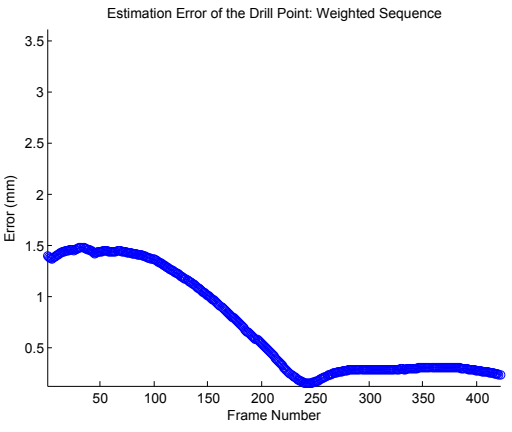

(b)

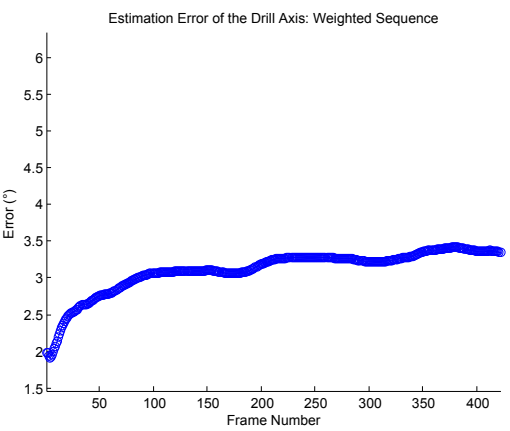

(d)

Fig. 2. Position error of the drill tip, (a) and (b), and angular error of the direction of the instrument axis, (c) and (d). In (a) and (c) each frame is considered individually. In (b) and (d), the error for the frame number $i$ is calculated using the weighted average over the frame sequence from 1 to $i$. 
Fig. 3. The result of the estimation of the position of the instrument tip and of the direction of the instrument axis are depicted in green. The reference position and orientation are depicted in red.

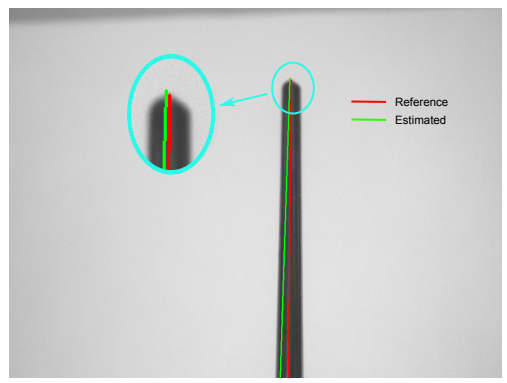

tracking systems usually require about 2 minutes for instrument calibration. In our setup, the calibration required about 20 seconds. Our experiments showed that using the whole sequence of acquired camera images contributes to obtain a more stable result that is less dependent from small axis oscillations which could occur when the instrument is in place in the calibration plate. Moreover, we showed that we can exploit the fact that the instrument axis position in the camera image does not change in order to obtain an error measure for the accuracy of our calibration. We expect to obtain a better accuracy in the direction estimation modifying our calibration plate in order to improve the stability of the instrument when placed on the plate, since this would allow a further reduction of the oscillation of the instrument axis. In future work, we aim to reduce the angular error of the instrument axis estimation to a value below $1^{\circ}$.

\section{References}

1. Nagel M, Schmidt G, Petzold R, Kalender W. A navigation system for minimally invasive CT-guided interventions. Medical Image Computing and Computer-Assisted Intervention-MICCAI. 2005; p. 33-40.

2. Reaungamornrat S, Otake Y, Uneri A, Schafer S, Mirota D, Nithiananthan S, et al. Tracker-on-C for cone-beam CT-guided surgery: evaluation of geometric accuracy and clinical applications. Proc SPIE Medical Imaging: Image-Guided Procedures, Robotic Interventions, and Modeling. 2012;8316.

3. Diotte B, Fallavollita P, Wang L, Weidert S, Thaller PH, Euler E, et al. RadiationFree Drill Guidance in Interlocking of Intramedullary Nails. Medical Image Computing and Computer-Assisted Intervention-MICCAI. 2012; p. 18-25.

4. Forman C, Aksoy M, Hornegger J, Bammer R. Self-encoded marker for optical prospective head motion correction in MRI. Medical Image Computing and Computer-Assisted Intervention-MICCAI. 2010; p. 259-266.

5. Schweighofer G, Pinz A. Robust pose estimation from a planar target. Pattern Analysis and Machine Intelligence, IEEE Transactions on. 2006;28(12):2024-2030.

6. Ma Y, Soatto S, Kosecka J, Sastry SS. An invitation to 3-D vision: from images to geometric models. vol. 26. Springer; 2004. 